\title{
The Relationship Between Systemic Immune Inflammation Index and Treatment Response in Renal Cell Carcinoma Patients Treated with Tyrosine Kinase Inhibitors: Results from The Turkish Oncology Group Kidney Cancer Consortium Database
}

Kadriye Bir Yücel

Ankara University

Emre Yekedüz

Ankara University

Serdar Karakaya University of Health Sciences Dr. Abdurrahman Yurtaslan Ankara Oncology Education and Research Hospital

\section{Deniz Tural}

University of Health Sciences, Bakirköy Dr. Sadi Konuk Training and Research Hospital İsmail Ertürk

University of Health Sciences, Gülhane Education and Research Hospital

Cihan Erol

Ankara Yıldırım Beyazıt University

Özlem Ercelep

Marmara University

Nihan Şentürk Öztaş

İstanbul University-Cerrahpaşa

Çağatay Arslan

İzmir University of Economics

Gökhan Uçar

University of Health Sciences, Ankara City Hospital

Ahmet Küçükarda

Trakya University

Özlem Nuray Sever

Gaziantep University

Saadettin Kılıçkap

İstinye University 


\section{Orçun Can}

University of Health Sciences, Prof. Dr. Cemil Taşçıoğlu City Hospital

\section{Satı Coşkun Yazgan}

Ankara University

\section{Berna Öksüzoğlu}

University of Health Sciences Dr. Abdurrahman Yurtaslan Ankara Oncology Education and Research Hospital

\section{Nuri Karadurmuş}

University of Health Sciences, Gülhane Education and Research Hospital

\section{Mehmet Ali Şendur}

Ankara Yıldırım Beyazıt University

Yüksel Ürün ( $\square$ yukselurun@gmail.com )

Ankara University

\section{Research Article}

Keywords: systemic immune inflammation index, renal cell cancer, prognosis, tyrosine kinase inhibitor Posted Date: December 6th, 2021

DOI: https://doi.org/10.21203/rs.3.rs-1101225/v1

License: (c) (i) This work is licensed under a Creative Commons Attribution 4.0 International License. Read Full License 


\section{Abstract}

Aim: To investigate the prognostic value of the systemic immune-inflammation index (SII) and its impact on survival in patients with metastatic renal cell carcinoma (mRCC).

Methods: A total of 706 patients with mRCC treated with tyrosine kinase inhibitors (TKIs) (i.e., sunitinib, pazopanib) were retrospectively analyzed. All patients were classified into either a high-SIl group or a lowSII group based on the cut-off value of SII at 756, which was the median SII level of our study group.

Results: The median age of patients was 60 (interquartile range (IQR):53-67) years. Three out of four patients were male. The majority of patients $(85.7 \%)$ had clear cell histology, and sarcomatoid differentiation was observed in $16.9 \%$ of all patients. The rates of patients in favorable, intermediate, and poor "International mRCC Database Consortium (IMDC)" risk groups were 20.1\%, 57.6\%, and 22.2\%, respectively. There were 311 and 310 patients in the SII-low and SII-high groups, respectively. At the median of 48.6 months follow-up, the median overall survival (OS) was 34.6 months and 14.5 months in the low- and high-SII groups, respectively $(p<0.001)$. In multivariate analysis, SII was an independent prognostic factor for OS (hazard ratio (HR):1.39, 95\% confidence interval $(\mathrm{Cl}): 1.05-1.85, \mathrm{p}=0.01$ ) and PFS (HR:1.60, 95\% Cl:1.24-2.05, p<0.001).

Conclusion: Pre-treatment level of high SIl might be considered as a predictor of poor prognosis in patients with $\mathrm{mRCC}$ treated with TKIs.

\section{Introduction}

Renal cell carcinoma (RCC) accounts for $90-95 \%$ of all kidney cancers. In 2020 , about $3 \%$ of all adult malignancies with an estimated 431,288 new RCC cases were observed across the world ${ }^{1,2}$. More than $30 \%$ of patients diagnosed with RCC need systemic therapy for metastatic disease ${ }^{3}$. Historically, treatment of metastatic RCC (mRCC) had been limited to cytokine therapies (i.e., interleukin-2 and interferon). However, tyrosine kinase inhibitors (TKIs) and mammalian target of rapamycin (mTOR) inhibitors improved the prognosis of those patients. Furthermore, immune checkpoint inhibitors (ICls) and ICls plus TKIs combinations initiated a new era in the treatment of $\operatorname{mRCC}{ }^{4,5}$.

In parallel to the improvements in the treatment of $\mathrm{mRCC}$, prognostic risk tools became essential during the decision-making process in the treatment of mRCC patients. Thus, the International Metastatic RCC Database (IMDC) risk model is the standard for prognostic stratification of patients with mRCC treated with targeted therapies or ICls ${ }^{6,7}$. The IMDC risk score is calculated by the following six parameters: Karnofsky performance status, time from diagnosis to the first systemic treatment, hemoglobin concentration, neutrophils, platelets, and corrected calcium levels.

Inflammatory-related peripheral cells (e.g., neutrophils, lymphocytes, platelets) derived from the peripheral blood were associated with tumor progression in various tumors. The prognostic significance of inflammatory cell parameters, such as neutrophil-lymphocyte ratio (NLR), platelet-lymphocyte ratio (PLR), 
C-reactive protein/albumin ratio, and systemic immune inflammation index (SII), were examined in many cancer types over the last ten years ${ }^{8-15}$. SII is a combination based on the peripheral lymphocyte, neutrophil, and platelet counts. After Hu et al. showed its prognostic value in 2014, a huge number of studies established that SII could be a good prognostic marker in many cancer types ${ }^{8}$.In this retrospective analysis, we aimed to evaluate the prognostic significance of SII in patients with mRCC treated with TKIs.

\section{Methods}

This retrospective cohort study was approved by the local ethical committee (Ankara University Faculty of Medicine Human Research Ethics Committee) and conducted in compliance with the "Declaration of Helsinki". Informed consent was waived by "Ankara University Faculty of Medicine Human Research Ethics Committee" due to the retrospective nature of the study.

\section{Patient Population and Data Extraction}

The Turkish Oncology Group Kidney Cancer Consortium (TKCC) database consists of approximately 1,000 patients aged 18 years and older with mRCC from 13 cancer centers in Turkey. Patients with mRCC treated with sunitinib or pazopanib in the first-line setting were extracted from the TKCC database.

Demographic data (e.g., date of birth, gender, comorbidities, medications), date of diagnosis with RCC, the initial date of systemic treatment in the metastatic setting, Eastern Cooperative Oncology Group (ECOG) performance score, laboratory findings (e.g., neutrophil, platelet, lymphocyte counts, hemoglobin concentration, corrected calcium level), start and end dates of TKIs, and dates of progression and death were extracted from the TKCC database.

SIl was calculated by using the following formula: [neutrophil (cells $\times 10^{9} / \mathrm{L}$ ) x platelet (cells $10^{9} / \mathrm{L}$ )] / lymphocyte (cells $\times 10^{9} / \mathrm{L}$ ). All values were obtained from a complete blood count (CBC) up to 30 days prior to the first dose of TKIs. If there were more than one $\mathrm{CBC}$ result, the closest one to the initiation of TKI was used. The best cut-off value for SII was determined by using the median value of 756 . With this regard, patients were divided into two groups as SII-high $(\geq 756)$ and SII-low $(<756)$. The primary outcome was overall survival (OS), and the secondary outcome was progression-free survival (PFS).

\section{Statistical Analyses}

To summarize data, median with interquartile range (IQR) or mean with standard deviation and percentages were used for continuous and categorical variables, respectively. The independent sample $t$ test or Mann-Whitney $U$ and chi-square tests were performed to compare continuous and categorical variables, respectively. Survival curves were estimated using the Kaplan-Meier method, and the differences between groups were analyzed by using the log-rank test. Cox proportional hazards regression model was used for multivariate analyses of parameters associated with OS and PFS. OS was calculated from the initial date of TKIs to death. PFS was calculated from the initial date of TKIs to disease progression or death. Hazard ratio (HR) and 95\% confidence interval (Cl) were used to describe 
the risk factors. Differences were considered significant if the p-value was less than 0.05 . All statistical analyses were performed using the SPSS 27.0 for Mac (IBM Corp., Armonk, NY).

\section{Results}

\section{Baseline Characteristics}

A total of 706 patients with mRCC were included in this study and SII was calculated in 621 patients. The median age of patients was 60 (IQR:53-67) years. Three out of four patients were male. The majority of patients $(85.7 \%)$ had clear cell histology, and $16.9 \%$ of all patients had sarcomatoid differentiation. The ECOG PS was 0 or 1 in most patients (83.5\%). Approximately one out of four patients were in the IMDC poor-risk group. In total, $404(57.2 \%)$ and $302(42.8 \%)$ patients were treated with sunitinib and pazopanib, respectively. Approximately half of the patients received interferon before TKI treatment. About three out of four patients underwent nephrectomy prior to the start of systemic treatment. Lung was the most common metastatic site (51.4\%).

There were 311 and 310 patients in the SII-low and SII-high groups, respectively. The rate of patients who underwent nephrectomy was higher in the SII-low group than in the SII-high group (83.9\% vs. $64.4 \%$, $p<0.001)$. Similarly, the rate of patients treated with sunitinib was higher in the SIl-low group than in the SII-high group ( $63.3 \%$ vs. $49.0 \%, p<0.001)$. As expected, the IMDC poor-risk patients' rate was higher in the SII-high group than in the SII-low group $(34.6 \%$ vs. $8.8 \%, p<0.001)$. All baseline characteristics of the included patients are shown in Table 1. 
Table 1

Baseline Characteristics

\begin{tabular}{|c|c|c|c|c|c|c|}
\hline & \multicolumn{2}{|c|}{ All Patients } & \multicolumn{2}{|c|}{ SII-Low Patients } & \multicolumn{2}{|c|}{ SII-High Patients } \\
\hline & $n=706$ & (\%) & $n=311$ & $(\%)$ & $n=310$ & (\%) \\
\hline Age-years, median (IQR) & \multicolumn{2}{|l|}{$60(53-67)$} & \multicolumn{2}{|l|}{$60(53-69)$} & \multicolumn{2}{|l|}{$60(53-70)$} \\
\hline \multicolumn{7}{|l|}{ Sex } \\
\hline Male & 531 & 75.2 & 229 & 73.6 & 239 & 77.1 \\
\hline Female & 175 & 24.8 & 82 & 26.4 & 71 & 22.9 \\
\hline \multicolumn{7}{|l|}{ Histological Type } \\
\hline Clear Cell & 563 & 79.7 & 241 & 77.5 & 257 & 82.9 \\
\hline Non-clear Cell & 94 & 13.3 & 46 & 14.8 & 36 & 11.6 \\
\hline Missing & 49 & 6.9 & 24 & 7.7 & 17 & 5.5 \\
\hline \multicolumn{7}{|l|}{ Sarcomatoid Feature } \\
\hline Yes & 83 & 11.8 & 35 & 11.3 & 39 & 12.6 \\
\hline No & 407 & 57.6 & 182 & 58.5 & 192 & 61.9 \\
\hline Missing & 216 & 30.6 & 94 & 30.2 & 79 & 25.5 \\
\hline \multicolumn{7}{|l|}{ Fuhrman Grade } \\
\hline $1-2$ & 124 & 17.6 & 63 & 20.3 & 43 & 13.9 \\
\hline $3-4$ & 297 & 42.1 & 129 & 41.4 & 133 & 42.9 \\
\hline Missing & 285 & 40.4 & 119 & 38.3 & 134 & 43.2 \\
\hline \multicolumn{7}{|l|}{ Previous Nephrectomy } \\
\hline Yes & 525 & 74.4 & 260 & 83.6 & 199 & 64.2 \\
\hline No & 177 & 25.1 & 50 & 16.1 & 110 & 35.5 \\
\hline Missing & 4 & 0.6 & 1 & 0.3 & 1 & 0.3 \\
\hline \multicolumn{7}{|l|}{ Systemic Treatment } \\
\hline Sunitinib & 404 & 57.2 & 197 & 63.3 & 152 & 49.0 \\
\hline Pazopanib & 302 & 42.8 & 114 & 36.7 & 158 & 51.0 \\
\hline \multicolumn{7}{|l|}{ IMDC Risk } \\
\hline Favorable & 116 & 16.4 & 83 & 26.7 & 33 & 10.6 \\
\hline Intermediate & 332 & 47.0 & 175 & 56.3 & 152 & 49.0 \\
\hline
\end{tabular}




\begin{tabular}{|c|c|c|c|c|c|c|}
\hline & \multicolumn{2}{|c|}{ All Patients } & \multicolumn{2}{|c|}{ SII-Low Patients } & \multicolumn{2}{|c|}{ SII-High Patients } \\
\hline & $n=706$ & (\%) & $n=311$ & $(\%)$ & $n=310$ & $(\%)$ \\
\hline Poor & 128 & 18.1 & 25 & 8.0 & 98 & 31.6 \\
\hline Missing & 130 & 18.4 & 28 & 9.0 & 27 & 8.7 \\
\hline \multicolumn{7}{|l|}{ MSKCC Risk } \\
\hline Favorable & 91 & 12.9 & 64 & 20.6 & 27 & 8.7 \\
\hline Intermediate & 279 & 39.5 & 148 & 47.6 & 128 & 41.3 \\
\hline High & 87 & 12.3 & 27 & 8.7 & 59 & 19.0 \\
\hline Missing & 249 & 35.3 & 72 & 23.2 & 96 & 31.0 \\
\hline \multicolumn{7}{|c|}{ Previous Cytokine Use } \\
\hline Yes & 334 & 47.3 & 152 & 48.9 & 125 & 40.3 \\
\hline No & 372 & 52.7 & 159 & 51.1 & 185 & 59.7 \\
\hline \multicolumn{7}{|l|}{ Metastatic Sites } \\
\hline Lung & 319 & 51.4 & 161 & 51.8 & 158 & 51.0 \\
\hline Bone & 259 & 41.7 & 100 & 32.2 & 159 & 51.3 \\
\hline Liver & 92 & 14.8 & 42 & 13.5 & 50 & 16.1 \\
\hline CNS & 58 & 9.3 & 18 & 5.8 & 40 & 12.9 \\
\hline \multicolumn{7}{|l|}{ Performance Status } \\
\hline ECOG 0-1 & 515 & 72.9 & 243 & 78.1 & 207 & 66.8 \\
\hline ECOG 2-3-4 & 149 & 21.1 & 55 & 17.7 & 87 & 28.1 \\
\hline Missing & 42 & 5.9 & 13 & 4.2 & 16 & 5.2 \\
\hline $\begin{array}{l}\text { Abbreviations: ECO } \\
\text { Carcinoma Databa } \\
\text { Center }\end{array}$ & $\begin{array}{l}\text { operative } \\
, I Q R=\ln t\end{array}$ & $\begin{array}{l}\text { cology } \\
\text { uartile }\end{array}$ & $\begin{array}{l}\text { roup, IML } \\
\text { nge, MSk }\end{array}$ & $\begin{array}{l}\text { ternati } \\
\text { Memol }\end{array}$ & $\begin{array}{l}\text { Metastc } \\
\text { Sloan Ket }\end{array}$ & $\begin{array}{l}\text { enal Ce } \\
\text { y Cance }\end{array}$ \\
\hline
\end{tabular}

\section{Survival Outcomes}

At the median follow-up of 48.6 months, the median OS and PFS were 26.1 months (95\% Cl: $22.5-29.7$ ) and 11.9 months (95\% Cl: 10.5-13.3), respectively. The median OS was longer in the SII-low group than in the SII-high group (34.6 months vs. 14.5 months, $p<0.001)$. Similarly, the median PFS was longer in the SII-low group than in the SII-high group (18.0 months vs. 7.7 months, $p<0.001)$. Kaplan-Meier estimates of OS and PFS are shown in Figure 1 and Figure 2. 
In subgroup analysis, the median OS was shorter in patients with older age ( $\geq 65)(p=0.003)$, sarcomatoid feature $(p=0.01)$, anemia $(p<0.001)$, hypercalcemia $(p<0.001)$, increased lactate dehydrogenase $(L D H)(p<0.001)$, and with a systemic treatment initiation interval of less than one year $(p<0.001)$. The median PFS was shorter in patients with anemia $(p<0.001)$, hypercalcemia $(p=0.001)$, increased LDH $(p=0.01)$, a shorter time to systemic treatment $(p<0.001)$, and history of cytokine use $(p<0.001)$. Conversely, the median OS and PFS were longer in patients with ECOG PS 0 or $1(p<0.001$ for OS and PFS) and in those who underwent nephrectomy ( $p<0.001$ for OS and PFS). The median OS was longer in the SII-low group than in the SII-high group irrespective of their previous interferon use (36.4 months vs. 16.6 months, $p=0.001$ in patients previously untreated with interferon; 30.1 months vs. 10.7 months, $p<0.001$ in patients previously treated with interferon). Similarly, the median PFS was also longer in the SII-low group than in the SII-high group irrespective of their previous interferon use (19.7 months vs. 8.1 months, $p<0.001$ in patients previously untreated with interferon; 15.7 months vs. 7.3 months, $p<0.001$ in patients previously treated with interferon). (Figure S1 and Figure S2)

After adjusting for confounding factors (i.e., age, sarcomatoid feature, nephrectomy, systemic treatment with sunitinib or pazopanib, anemia, hypercalcemia, LDH elevation, ECOG PS, time from diagnosis to systemic treatment for OS; age, sarcomatoid feature, nephrectomy, anemia, hypercalcemia, LDH elevation, ECOG PS, time from diagnosis to systemic treatment for PFS), SIl was an independent prognostic factor for OS (HR:1.39, 95\% Cl:1.05-1.85, p=0.01) and PFS (HR:1.60, 95\% Cl:1.24-2.05, $\mathrm{p}<0.001)$.

\section{Discussion}

In this multicenter study, we investigated the prognostic value of SII in patients with mRCC treated with TKIs. The results showed that low $(<756)$ and high $(\geq 756)$ SIl levels had a statistically significant difference in terms of OS. Thus, SII might have a prognostic value in patients with mRCC treated with TKIs.

The relationship between inflammation and cancer has been widely investigated in many previous studies. Inflammatory cells (e.g., neutrophils, macrophages, lymphocytes ) and cytokines are effective on transformation, proliferation, and metastasis in all tumor stages ${ }^{16}$. Neutrophils can secrete cytokines related to the stimulation of the tumor microenvironment and have a tumor-promoting activity, including cancer cell survival and proliferation, angiogenesis, and metastasis ${ }^{13}$. Conversely, lymphocytes inhibit tumor cell proliferation by secreting cytokines. On the other hand, platelets regulate cancer invasion, migration, and angiogenesis by secretion of numerous chemokines and growth factors ${ }^{17}$.

Numerous studies revealed the impact of prognostic nomograms based on peripheral inflammatory cells. With this regard, one of the most investigated prognostic markers is the neutrophil-to-lymphocyte ratio (NLR). It was associated with the prognosis in most tumors such as RCC, lung, colorectal, urothelial, and pancreatic cancers ${ }^{14,15,18-24}$. 
SIl is a marker of inflammation and immunity based on neutrophil, lymphocyte, and platelet counts, which are relatively easy to obtain in clinical practice. In 2014, Hu et al. developed SIl to predict the prognosis of patients who underwent curative resection for hepatocellular carcinoma and established that a high SII score $\left(>330 \times 10^{9}\right.$ cells/L) indicated a poor outcome in those patients ${ }^{8}$. Subsequently, SII has been investigated as a marker to predict cancer survival in various tumors, such as gastric cancer, germ-cell tumor, and prostate cancer ${ }^{[6-12]}$. To the best of our knowledge, one study evaluated the relationship between SII and the prognosis in patients with $\mathrm{mRCC}$ treated with targeted therapy. In that study, Lolli et al. showed that low SII was associated with poorer survival in 335 patients with mRCC treated with sunitinib ${ }^{25}$. In this context, our results are consistent with the literature. Furthermore, in the study of Lolli et al., the pre-treatment SII cut-off value was determined as $730 \times 10^{9}$ cells $/ \mathrm{L}$, which was numerically close to our study's SII cut-off value. It should be noticed that number of patients was higher in our study. In addition, while only sunitinib was administered as a first-line treatment in the study of Lolli et al., patients who received sunitinib or pazopanib in our study. However, patients with mRCC who received interferon before TKIs were also included in our study.

Of note, our findings were also consistent with the literature according to IMDC risk groups. With regard to the prognostic value of IMDC risk score and SII combination, Chrom et al. showed that replacement of neutrophil and platelet counts with SII in the IMDC risk model increased the accuracy of the IMDC risk model. It should be noticed that they also used a cut-off value of $730 \times 10^{9}$ cells $/ \mathrm{L}$ for SIl, which is almost the same as our study 26 .

Our survival results were also compatible with the pivotal study of sunitinib, including previously untreated patients with mRCC. They reported that the median OS was 26.4 months and PFS was 11 months in patients with mRCC receiving sunitinib, which was also numerically close to our study's survival results. ${ }^{27}$

Our study has several limitations due to its retrospective nature. First, we had a lack of data to calculate SIl in some patients. Because of this reason, we had to exclude those patients from our study. Second, the time interval between obtaining laboratory values to calculate SII and the initial date of TKIs might be different in each included center. Third, mRCC patients treated with interferon before TKI treatment were included in our study.

In conclusion, our study showed the prognostic value of SII in mRCC patients treated with TKIs. In this context, SII, easily accessible marker, might lead to the establishment of novel therapeutic strategies or risk models in $\mathrm{MRCC}$ patients treated with TKIs. To the best of our knowledge, the relationship of ICls plus TKIs combinations with SII has not been investigated yet. From a future perspective, SII may be a potential prognostic marker for RCC patients treated with immunotherapy.

\section{Declarations}

\section{Authors' Contribution}


Conceptualization: KBY, EY, SK, DT, IE, CE, ÖE, NŞÖ, ÇA, GU, AK, ÖNS, SK, OÇ, SCY, BÖ, NK, MAŞ, YÜ

Methodology: KBY, EY, YÜ

Software: EY

Data Curation: KBY, EY, SK, DT, IE, CE, ÖE, NŞÖ, ÇA, GU, AK, ÖNS, SK, OÇ, SCY, BÖ, NK, MAŞ, YÜ

Writing - Original Draft: KBY, EY

Writing - Review \& Editing: KBY, EY, SK, DT, IE, CE, ÖE, NŞÖ, ÇA, GU, AK, ÖNS, SK, OÇ, SCY, BÖ, NK, MAŞ, YÜ

Supervision: SK, DT, IE, CE, ÖE, NŞÖ, ÇA, GU, AK, ÖNS, SK, OÇ, SCY, BÖ, NK, MAŞ, YÜ

\section{Declaration of conflicting interests}

The author(s) report no competing personal or financial interests related to this article.

\section{Funding.}

No financial supports for author(s) to declare

\section{References}

1 Sung, H. et al. Global cancer statistics 2020: GLOBOCAN estimates of incidence and mortality worldwide for 36 cancers in 185 countries. CA: a cancer journal for clinicians (2021).

2 Siegel, R. L., Miller, K. D. \& Jemal, A. Cancer statistics, 2019. CA: a cancer journal for clinicians 69, 7-34 (2019).

3 Lam, J. S., Shvarts, O., Leppert, J. T., Figlin, R. A. \& Belldegrun, A. S. Renal cell carcinoma 2005: new frontiers in staging, prognostication and targeted molecular therapy. The Journal of urology 173, 1853-1862 (2005).

$4 \quad$ Tannir, N. M. et al. (American Society of Clinical Oncology, 2019).

5 Rini, B. I. et al. Pembrolizumab plus axitinib versus sunitinib for advanced renal-cell carcinoma. New England Journal of Medicine 380, 1116-1127 (2019).

6 Motzer, R. J. et al. Survival and prognostic stratification of 670 patients with advanced renal cell carcinoma. Journal of clinical oncology 17, 2530-2530 (1999).

$7 \quad$ Heng, D. Y. et al. Prognostic factors for overall survival in patients with metastatic renal cell carcinoma treated with vascular endothelial growth factor-targeted agents: results from a large, multicenter study. Journal of clinical oncology 27, 5794-5799 (2009). 

resection for hepatocellular carcinoma. Clinical Cancer Research 20, 6212-6222 (2014).

9 Zhong, J.-H., Huang, D.-H. \& Chen, Z.-Y. Prognostic role of systemic immune-inflammation index in solid tumors: a systematic review and meta-analysis. Oncotarget 8, 75381 (2017).

10 Chen, J.-H. et al. Systemic immune-inflammation index for predicting prognosis of colorectal cancer. World journal of gastroenterology 23, 6261 (2017).

11 Tong, Y.-S., Tan, J., Zhou, X.-L., Song, Y.-Q. \& Song, Y.-J. Systemic immune-inflammation index predicting chemoradiation resistance and poor outcome in patients with stage III non-small cell lung cancer. Journal of translational medicine 15, 1-10 (2017).

12 Lolli, C. et al. Systemic immune-inflammation index predicts the clinical outcome in patients with mCRPC treated with abiraterone. Frontiers in pharmacology 7, 376 (2016).

13 Templeton, A. J. et al. Prognostic role of neutrophil-to-lymphocyte ratio in solid tumors: a systematic review and meta-analysis. JNCl: Journal of the National Cancer Institute 106 (2014).

14 Rossi, L. et al. High neutrophil-to-lymphocyte ratio persistent during first-line chemotherapy predicts poor clinical outcome in patients with advanced urothelial cancer. Annals of surgical oncology 22, 1377-1384 (2015).

15 Templeton, A. J. et al. Change in neutrophil-to-lymphocyte ratio in response to targeted therapy for metastatic renal cell carcinoma as a prognosticator and biomarker of efficacy. European urology 70 , 358-364 (2016).

16 Mantovani, A., Allavena, P., Sica, A. \& Balkwill, F. Cancer-related inflammation. nature 454, 436444 (2008).

17 Schumacher, D., Strilic, B., Sivaraj, K. K., Wettschureck, N. \& Offermanns, S. Platelet-derived nucleotides promote tumor-cell transendothelial migration and metastasis via $\mathrm{P} 2 \mathrm{Y} 2$ receptor. Cancer cell 24, 130-137 (2013).

18 Nunno, V. D. et al. Prognostic impact of neutrophil-to-lymphocyte ratio in renal cell carcinoma: a systematic review and meta-analysis. Immunotherapy 11, 631-643 (2019).

19 Teishima, J. et al. Impact of neutrophil-to-lymphocyte ratio on effects of targeted therapy for metastatic renal cell carcinoma patients with extrapulmonary metastasis. Canadian Urological Association Journal 11, E207 (2017).

20 Huszno, J., Kolosza, Z., Mrochem-Kwarciak, J., Rutkowski, T. \& Skladowski, K. The role of neutrophil-lymphocyte ratio, platelet-lymphocyte ratio, and platelets in the prognosis of metastatic renal cell carcinoma. Oncology 97, 7-17 (2019). 
21 Tanaka, N. et al. in Urologic Oncology: Seminars and Original Investigations. 39. e19-39. e28 (Elsevier).

22 Diem, S. et al. Neutrophil-to-Lymphocyte ratio (NLR) and Platelet-to-Lymphocyte ratio (PLR) as prognostic markers in patients with non-small cell lung cancer (NSCLC) treated with nivolumab. Lung cancer 111, 176-181 (2017).

23 An, X. et al. Elevated neutrophil to lymphocyte ratio predicts survival in advanced pancreatic cancer. Biomarkers 15, 516-522 (2010).

24 Walsh, S., Cook, E., Goulder, F., Justin, T. \& Keeling, N. Neutrophil-lymphocyte ratio as a prognostic factor in colorectal cancer. Journal of surgical oncology 91, 181-184 (2005).

25 Lolli, C. et al. Systemic immune-inflammation index predicts the clinical outcome in patients with metastatic renal cell cancer treated with sunitinib. Oncotarget 7, 54564 (2016).

26 Chrom, P., Zolnierek, J., Bodnar, L., Stec, R. \& Szczylik, C. External validation of the systemic immune-inflammation index as a prognostic factor in metastatic renal cell carcinoma and its implementation within the international metastatic renal cell carcinoma database consortium model. International journal of clinical oncology 24, 526-532 (2019).

27 Motzer, R. J. et al. Sunitinib versus interferon alfa in metastatic renal-cell carcinoma. N Eng/ J Med 356, 115-124, doi:10.1056/NEJMoa065044 (2007).

\section{Figures}




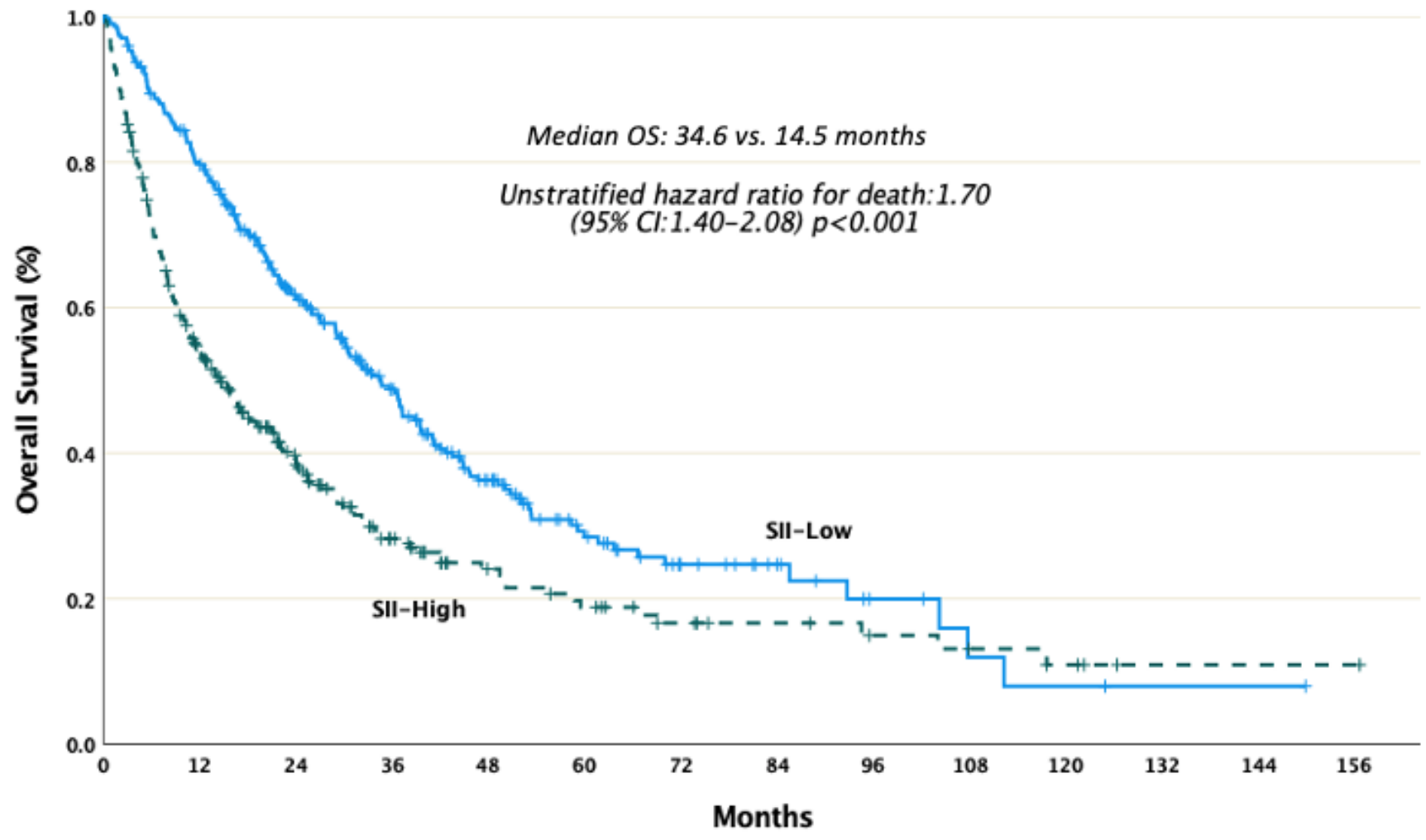

\section{Number at Risk:}

$\begin{array}{lllllllllllllll}\text { SII-Low } & 311 & 236 & 158 & 105 & 63 & 35 & 19 & 12 & 6 & 3 & 2 & 1 & 1 & 0 \\ \text { SII-High } & 310 & 152 & 88 & 48 & 28 & 21 & 14 & 11 & 8 & 6 & 4 & 1 & 1 & 1\end{array}$

Figure 1

Kaplan-Meier Estimates of Overall Survival (OS) (SII=Systemic Immune Inflammation Index) 


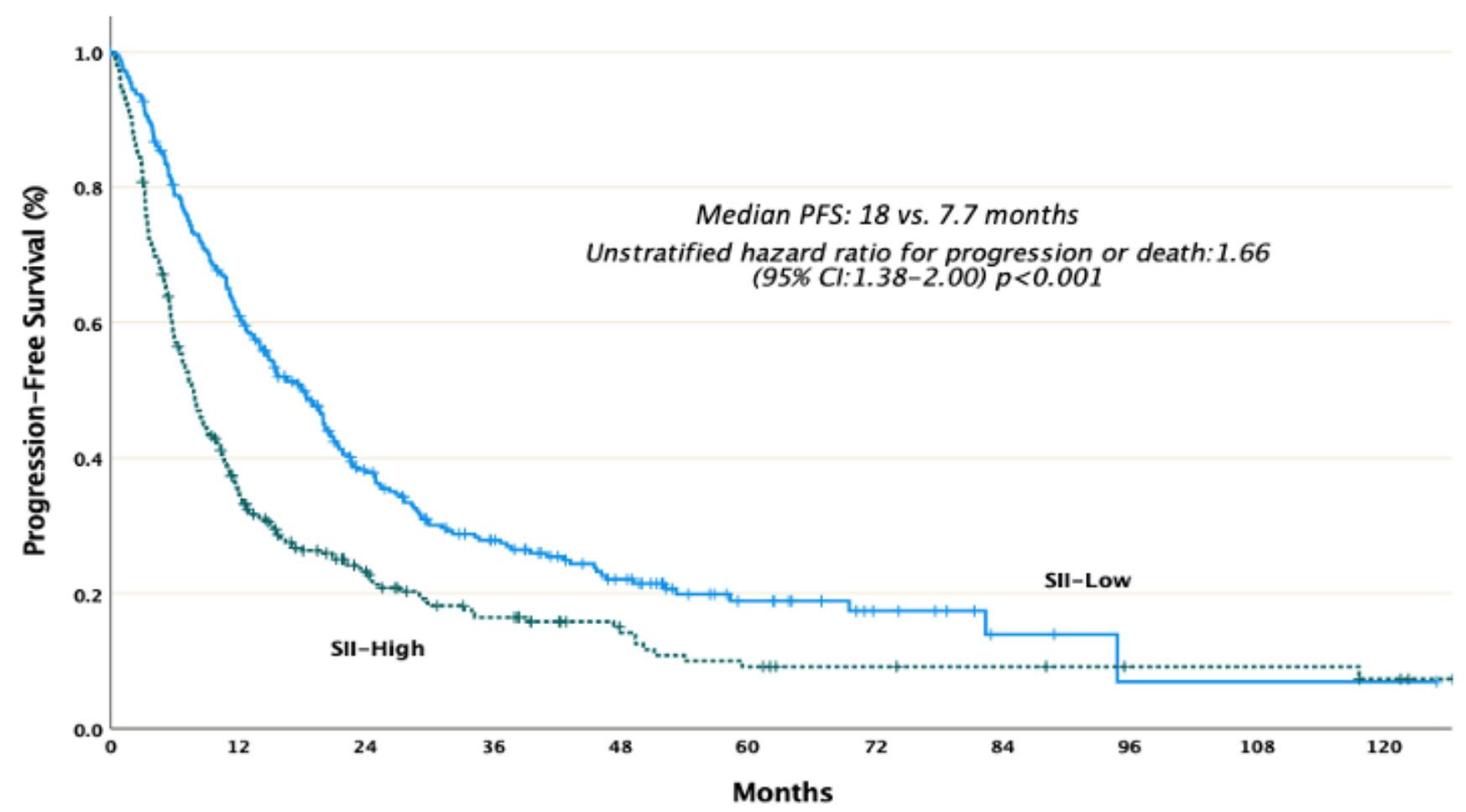

Number at Risk:

$\begin{array}{llllllllllll}\text { SII-Low } & 311 & 182 & 96 & 60 & 37 & 18 & 9 & 3 & 1 & 1 & 1 \\ \text { SII-High } & 310 & 100 & 50 & 29 & 18 & 11 & 8 & 7 & 5 & 5 & 3\end{array}$

Figure 2

Kaplan-Meier Estimates of Progression-Free Survival (PFS) (SII=Systemic Immune Inflammation Index)

\section{Supplementary Files}

This is a list of supplementary files associated with this preprint. Click to download.

- Supplementary.docx 